\title{
Ciclo gametogénico e índice de condición de la almeja Chione fluctifraga en Bahía San Jorge, Golfo de California, México
}

\author{
Gametogenic cycle and condition index of the clam Chione fluctifraga in \\ Bahia San Jorge, Gulf of California, Mexico

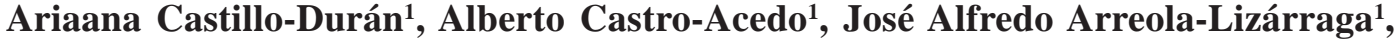 Francisco Hoyos-Chairez², Carlos Cáceres-Martínez ${ }^{3}$, Renato Mendoza-Salgado ${ }^{1}$, Luis Martínez-Córdova ${ }^{4}$ y Jorge Chávez-Villalba ${ }^{1}$

\begin{abstract}
${ }^{1}$ Centro de Investigaciones Biológicas del Noroeste (CIBNOR), Unidad Sonora, Apdo. Postal 349, Guaymas, Sonora 85454, México. jechavez04@cibnor.mx

${ }^{2}$ Instituto de Acuacultura, Estado de Sonora O.P.D., Comonfort y Av. de la Cultura, Hermosillo, Sonora 83280, México ${ }^{3}$ Universidad Autónoma de Baja California Sur, Apdo. Postal 19-B, La Paz, B.C.S. 23080, México ${ }^{4}$ Departamento de Investigaciones Científicas y Tecnológicas, Universidad de Sonora, Rosales y Niños Héroes, Apdo. Postal 1819, Hermosillo, Sonora, 83000, México
\end{abstract}

\begin{abstract}
The gametogenic cycle of the black clam Chione fluctifraga of northwestern Mexico, collected from March 2009 through February 2010, was studied using conventional histological techniques and oocyte modal analysis. A condition index was also determined. Clams exhibited a semi-annual gametogenic cycle. The species generated the first oocyte cohort in spring (April) started its vitellogenic growth and remained continuous over 9 months until early winter (December). A resting period occurs in winter. Clams released gametes continuously throughout the reproductive period, but the main spawning occurred during the warmest period $\left(>28^{\circ} \mathrm{C}\right)$. Gametogenic activity seemed to be strongly regulated by water temperature. The average growth rate of oocyte cohorts was calculated $\left(0.41 \pm 0.10 \mu \mathrm{m}\right.$ day $\left.{ }^{-1}\right)$, with the most rapid growth $\left(0.67 \mu \mathrm{m} \mathrm{day}{ }^{-1}\right)$ from September through October. The condition index was higher in summer, but other peaks occurred in winter, producing accumulation of nutrients from increased phytoplankton biomass. The female:male ratio was 1:1.3; no hermaphrodites were detected. A reproductive classification is proposed. These results can help regulate exploitation and establish off-limit periods for this clam in the Gulf of California.
\end{abstract}

Key words: Black clam, Chione fluctifraga, gametogenesis, spawning, oocyte stages, sex ratio

Resumen.- El ciclo gametogénico de la almeja negra Chione fluctifraga en el noroeste de México fue estudiado mediante técnicas histológicas convencionales y distribuciones de talla de ovocitos (análisis modal) durante marzo 2009-febrero 2010. Adicionalmente se determinó un índice de condición general. Las almejas presentaron un ciclo gametogénico semianual. La especie inició la formación de la primera cohorte ovocitaria en primavera (abril) comenzando así su crecimiento vitelogénico que se mantuvo continuo durante 9 meses hasta finales de otoño (diciembre), para posteriormente pasar a un periodo de reposo durante invierno. Las almejas liberaron constantemente gametos al medio pero presentaron los principales desoves durante el periodo más cálido $\left(>28^{\circ} \mathrm{C}\right)$. La actividad gametogénica pareció estar fuertemente controlada por la temperatura del agua. Se calculó la tasa promedio de crecimiento de las cohortes ovocitarias $\left(0,41 \pm 0,10 \mu \mathrm{m}\right.$ día $\left.^{-1}\right)$ registrándose un máximo de 0,67 $\mu \mathrm{m}$ día ${ }^{-1}$ entre septiembre-octubre. El índice de condición (IC) fue mayor en verano, aunque otro pico ocurrió en invierno produciéndose una acumulación de nutrientes a partir de un incremento de biomasa de fitoplancton. La proporción de sexos (hembra: macho) fue 1:1,3 y no se encontraron almejas hermafroditas. Se propone una escala reproductiva para la especie. Estos resultados pueden contribuir en las regulaciones de explotación y establecimiento de vedas biológicas del recurso en el Golfo de California.

Palabras clave: Almeja negra, Chione fluctifraga, gametogénesis, desoves, estadios ovocitarios, proporción sexos

\section{INTRODUCCIÓN}

La almeja negra Chione fluctifraga (Sowerby, 1853) es un molusco bivalvo que se distribuye en el sur de California (Estados Unidos), en la costa del Pacífico de la Península de Baja California y en el Golfo de California (Martínez-
Córdova 1996). Esta especie sostiene una pesquería de 1,600 ton anuales (Tinoco-Orta \& Cáceres-Martínez 2003), producción que se ha mantenido sin variación hasta la actualidad. La pesquería de esta almeja es el sustento de 
algunas comunidades en el Golfo de California, donde se extraen manualmente de sustratos limosos o de arena fina donde viven enterradas a profundidades de 10 a $20 \mathrm{~cm}$ (Martínez-Córdova 1988). La mayoría de la producción se envía a la región norte de la Península (Baja California, México) donde se limpia y se clasifica por tallas para ser exportada a los Estados Unidos de América. Existe un mercado regional pero el volumen de consumo representa el 5\% de la producción anual. A pesar de su importancia se carece de bases científicas para el manejo de esta pesquería. Las autoridades en materia de pesca requieren información sobre el ciclo de reproducción donde se determine con precisión los periodos de maduración y desove para el uso, manejo y preservación del recurso.

Existe poca información sobre esta almeja, pero hay estudios que describen aspectos reproductivos en otras especies del mismo género que se distribuyen en la misma región como Chione undatella (Baqueiro \& Masso 1988) y Chione californiensis (García-Domínguez 2002). Sólo hay un trabajo en condiciones de laboratorio sobre maduración gonádica de C. fluctifraga usando sistemas abiertos y cerrados de alimentación (Gutiérrez-Vázquez 2007), por lo que no existe información acerca de la biología reproductiva e índice de condición de la especie en poblaciones naturales.

La condición reproductiva de los bivalvos se determina por varias técnicas y en la mayoría de los trabajos se analizan preparaciones histológicas para conocer las características citológicas de las gónadas durante el proceso de gametogénesis (Gribben et al. 2004, Dutertre et al. 2009). Sin embargo, la observación de las laminillas histológicas puede resultar en interpretaciones subjetivas de la fase de reproducción en la que se encuentran los organismos en estudio (Barber \& Blake 1991). Para evitar este problema algunos autores señalan la importancia de combinar metodologías para obtener información cuantitativa, además de la cualitativa (Metaxatos 2004, Moura et al. 2008, Oyarzún-Cabañas et al. 2010). Entre los métodos cuantitativos más utilizados se encuentran el conteo y medición de los ovocitos (Muranaka \& Lannan 1984, Lango-Reynoso et al. 2000), así como el cálculo de áreas de la gónada (Heffernan \& Walker 1989, Enríquez-Díaz et al. 2009). Existen estudios donde los métodos anteriores se complementan analizando la distribución de las frecuencias de los diámetros de los ovocitos para conocer la evolución de las cohortes ovocitarias en el tiempo (Morvan \& Ansell 1988, LangoReynoso et al. 2006, Hmida et al. 2010). La información obtenida de esta manera permite determinar, teóricamente, el número de eventos de desove anual y la época de su ocurrencia. Esta información puede enriquecerse con la estimación del índice de condición (IC) gravimétrico para moluscos bivalvos (Crosby \& Gale 1990), ya que en algunos casos el IC se relaciona directamente con el ciclo reproductivo (Borda \& Cruz 2004, Lista et al. 2008, Sokolowski et al. 2010), o indica los periodos en donde la biomasa del individuo encuentra su mayor expresión (Lucas \& Beninger 1985).

Este estudio tuvo como objetivo describir el ciclo gametogénico de la almeja negra Chione fluctifraga de la población de Bahía San Jorge, Sonora, México, aplicando los métodos de estudio cualitativo y cuantitativo sobre los ovocitos de la especie. Asimismo, se determinaron las fases reproductivas en los machos y un índice de condición general.

\section{Materiales y Métodos}

\section{Muestreo}

El estudio se realizó en Bahía San Jorge (3101’54,47"N y $113^{\circ} 05^{\prime} 32,48$ "O), ubicada en la región noroeste de México. Los muestreos se efectuaron en la zona intermareal, donde se encuentran poblaciones naturales de Chione fluctifraga sujetas a explotación comercial (Fig. 1). Mensualmente, desde marzo 2009 hasta febrero 2010, se tomaron muestras de 60 almejas (35 a $55 \mathrm{~mm}$ altura de la concha), para ser transportadas al laboratorio del Centro de Investigaciones Biológicas del Noroeste en Guaymas, Sonora, localizado a $600 \mathrm{~km}$ del área de estudio. Las almejas fueron transportadas envueltas en papel húmedo dentro de una bolsa con hielo.

\section{TÉCNICA HISTOLÓGICA}

De cada muestra mensual de almejas, se utilizaron 15 individuos para los estudios histológicos. Para ello se obtuvo por disección una muestra de aproximadamente 1 $\mathrm{cm}^{-3}$ de la región media de la gónada de cada almeja. Las muestras se fijaron en solución Davidson durante 48 h y posteriormente fueron sometidas a un procesamiento histológico convencional; las muestras se deshidrataron usando una serie ascendente de concentraciones de etanol, posteriormente fueron aclaradas en xileno, luego se formaron bloques de parafina que incluían las gónadas, a los que se les realizaron cortes de $5 \mu \mathrm{m}$ de grosor usando un micrótomo marca Leica $\odot 82011$ y posteriormente teñirlos con hematoxilina-eosina (Howard \& Smith 1983). 
Figura 1. Sitio de explotación de la almeja negra Chione fluctifraga en Bahía San Jorge, en Sonora (México) / Production site of the black clam Chione fluctifraga at Bahía San Jorge in the State of Sonora (Mexico)

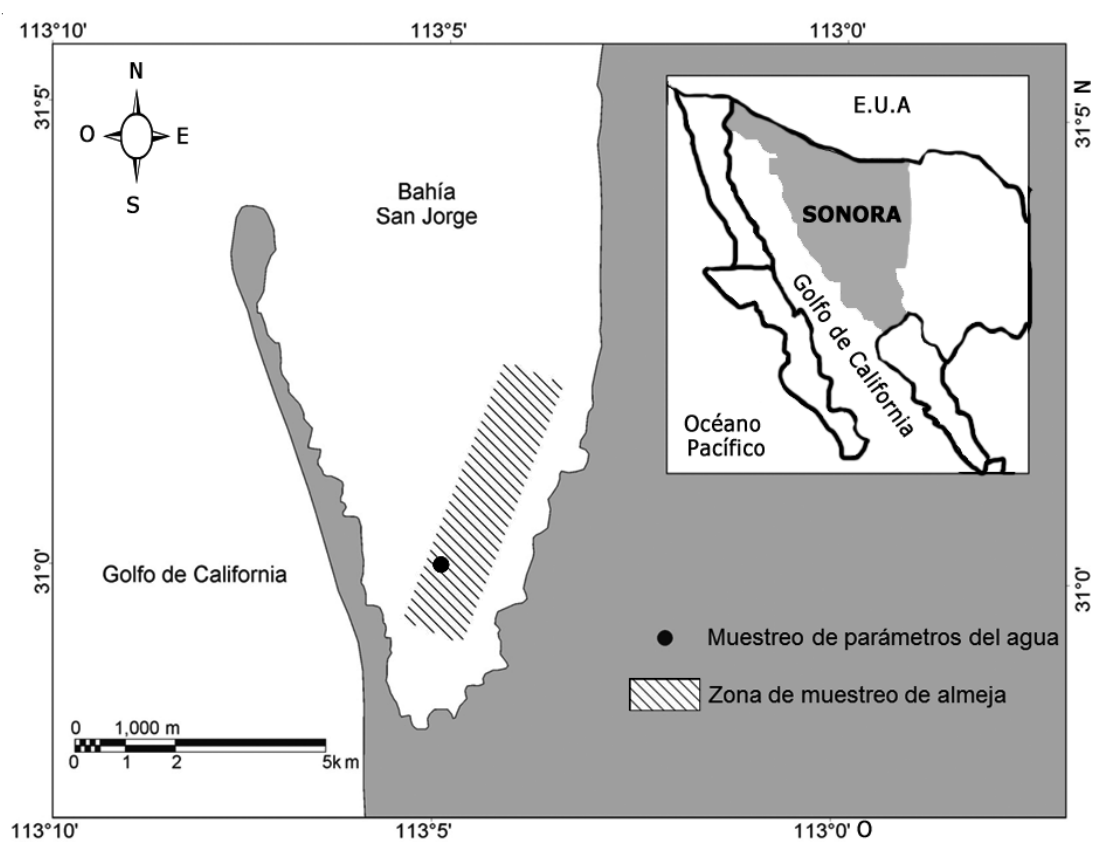

\section{Medición de OVocitos}

Con las preparaciones histológicas se identificó el sexo de los organismos por observación a través de un microscopio óptico Olympus $®$ BX41. De cada hembra se tomaron entre 5 a 10 imágenes digitales para así disponer de al menos 100 ovocitos por almeja para mediciones futuras. Sólo los ovocitos que presentaron una vesícula germinal bien definida, se les midió la superficie (pixeles) usando el software Sigma Scan Pro 5.0® y con ella se estimó el diámetro teórico (en $\mu \mathrm{m}$ ) mediante la relación propuesta por Lango-Reynoso et al. (2000):

$$
D_{\text {teórico }}=\sqrt{ } 4 \mathrm{~s} / \pi
$$

donde $D=$ diámetro y $S$ = superficie.

\section{ANÁLISIS MODAL}

Para determinar el total de ovocitos de cada almeja, se aplicó un análisis de progresión modal según el método de Bhattacharya (1967) usando el Software FAO-ICLARM Fish Stock Assessment Tools FiSAT II v. 1.2.1. Se obtuvieron los promedios y desviación estándar (DE) de las modas del diámetro de cada estado gametogénico, y las frecuencias correspondientes a cada mes de muestreo. Con estos resultados y las observaciones histológicas se asoció el intervalo de valores del diámetro ovocitario correspondiente a cada estadio de desarrollo gonádico, y se estableció el intervalo de valores del diámetro de los ovocitos correspondiente a cada estadio de desarrollo para esta especie.

Los diámetros de los ovocitos (previtelogénicos, vitelogénicos y maduros), obtenidos mensualmente fueron agrupados según los intervalos de desarrollo gonádico, se obtuvieron el promedio, valor máximo y mínimo, y fueron usados para obtener el intervalo de dispersión correspondiente. La información anterior fue usada para identificar gráficamente las cohortes ovocitarias (Fig. 2), y conocer el número de días transcurrido entre cada estadio identificado en el interior de cada cohorte. Esto permite conocer, teóricamente, la trayectoria que sigue en el tiempo un ovocito previtelogénico hasta llegar a ovocito maduro y ser desovado.

\section{Crecimiento de Los ovocitos}

Una vez que las cohortes ovocitarias fueron identificadas, se calculó la tasa promedio de crecimiento diaria de los ovocitos para cada una de ellas. Para ello se consideró la talla promedio de los ovocitos al inicio y al final del desarrollo de cada cohorte; al valor mayor se le restó el valor menor y el resultado se dividió por el número de días que tardó la cohorte en desarrollarse. Finalmente, se aplicó una regresión lineal simple y su correspondiente valor de correlación lineal de Pearson (Zar 1996). 


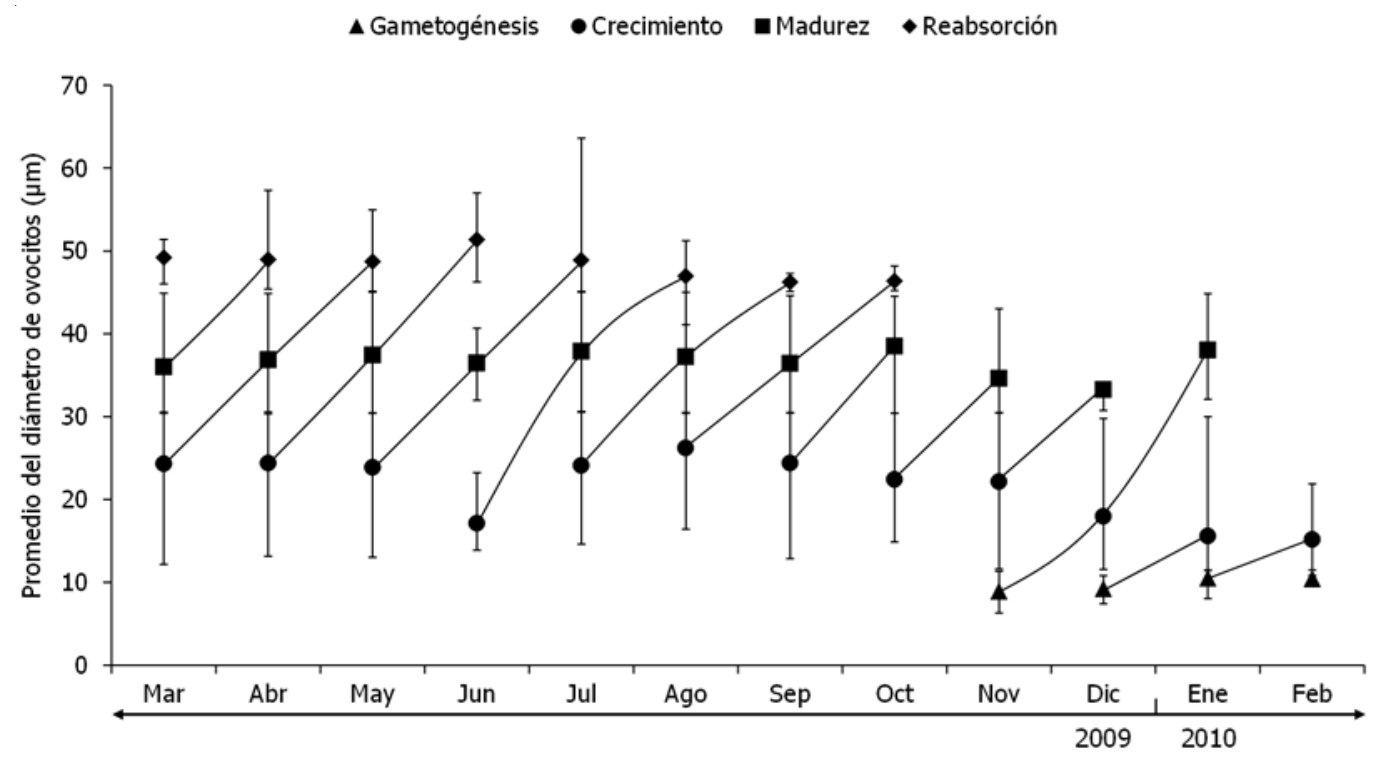

Figura 2. Resultados del análisis modal mostrando la evolución teórica de las cohortes ovocitarias de Chione fluctifraga / Results of modal analyses showing theoric evolution of the oocyte cohorts of Chione fluctifraga

\section{FASES REPRODUCTIVAS Y PROPORCIÓN DE SEXOS}

Para determinar las fases reproductivas de las hembras y machos de C. fluctifraga, se usaron como referencia los mismos criterios de clasificación (diámetro de ovocitos y análisis modal para hembras, y porcentaje de llenado de los folículos en machos) que en las escalas de reproducción propuestas por Lango-Reynoso et al. (2000) y Chávez-Villalba et al. (2002) para Crassostrea gigas. La prueba de Chi cuadrado se usó para determinar la bondad de ajuste de las proporciones sexuales con la relación esperada de 1:1.

\section{ÍNDICE DE CONDICIÓN}

Se utilizaron 45 individuos cada mes para calcular el índice de condición (IC) establecido por Walne \& Mann (1975)

$$
\text { IC }=\text { pt } 1000 / \text { pc }
$$

donde, IC es el índice de condición, pt: peso seco del tejido en gramos, y pc: peso seco de la concha en gramos.

\section{Parámetros ambientales}

En cada muestreo se registró la temperatura y salinidad del agua mediante un multisensor (YSI ${ }^{\circledR} 556 \mathrm{MPS}$ ). Al mismo tiempo se tomaron $2 \mathrm{~L}$ de agua para la determinación de clorofila $a$ mediante el método de espectrofotometría propuesto por Parsons et al. (1984); extracción con acetona de pigmentos de material fitoplanctónico retenido en filtros de fibra de vidrio Whatman ${ }^{\circledR}$ GF/C de $47 \mathrm{~mm}$ de diámetro de $1,2 \mu \mathrm{m}$ de abertura de poro. La determinación de seston se realizó usando la técnica descrita por Strickland \& Parson (1972) la cual consiste en filtrar un volumen de agua de mar $(1 \mathrm{~L})$ a través de filtros Whatman ${ }^{\circledR}$ GF/C con diámetro de 4,7 $\mathrm{cm}$. Los filtros se secan en un horno a $80^{\circ} \mathrm{C}$ durante $24 \mathrm{~h}$ y posteriormente se pesan para obtener el seston inorgánico. Los filtros se colocan después en una mufla por $4 \mathrm{~h}$ a $250^{\circ} \mathrm{C}$ para quemar el material orgánico y por diferencia obtener el seston orgánico.

\section{Resultados}

\section{ANÁLISIS MODAL}

El análisis modal permitió identificar 11 cohortes ovocitarias a lo largo del estudio que mostraron su correspondiente desove, desde marzo hasta enero; posteriormente, se identificaron 2 cohortes una en enero y otra en febrero 2010 que no se asocian con una evolución o crecimiento ovocitario del mes posterior (Fig. 2). Los estadios de desarrollo gonádico y los resultados del análisis modal sobre los diámetros de los ovocitos durante todo el estudio, permitieron asociar el intervalo de valores del diámetro correspondiente a cada estadio de desarrollo gonádico: inicio de gametogénesis $<12 \mu \mathrm{m}$, crecimiento 12,1-30 $\mu \mathrm{m}$, madurez 30,1-45 $\mu \mathrm{m}$, y reabsorción $>45 \mu \mathrm{m}$ (Tabla 1). 
Tabla 1. Resultado del análisis modal de los diámetros de los ovocitos de Chione fluctifraga. Se indica el valor promedio correspondiente a cada estadio de desarrollo gonádico / Modal analysis results of oocyte diameters of Chione fluctifraga. Values of mean diameters of each gonadal development stage are indicated

\begin{tabular}{|c|c|c|c|c|}
\hline Muestreo & Modas & $\begin{array}{c}\text { Promedio } \\
\text { correspondiente } \pm \sigma\end{array}$ & $\mathrm{n}$ & $\begin{array}{l}\text { Estadio de desarrollo } \\
\text { gonádico correspondiente }\end{array}$ \\
\hline \multirow[t]{2}{*}{ Mar-09 } & 1 & $15,9 \pm 3,9$ & 76 & Crecimiento \\
\hline & 2 & $30,2 \pm 4,3$ & 125 & Crecimiento \\
\hline \multirow[t]{2}{*}{ Abr-09 } & 1 & $24,1 \pm 7,0$ & 180 & Crecimiento \\
\hline & 2 & $35,3 \pm 6,8$ & 54 & Madurez \\
\hline \multirow[t]{2}{*}{ May-09 } & 1 & $19,9 \pm 5,6$ & 65 & Crecimiento \\
\hline & 2 & $35,0 \pm 7,0$ & 87 & Madurez \\
\hline \multirow[t]{3}{*}{ Jun-09 } & 1 & $12,0 \pm 2,9$ & 36 & Crecimiento \\
\hline & 2 & $30,0 \pm 6,0$ & 7 & Crecimiento \\
\hline & 3 & $47,9 \pm 6,2$ & 13 & Reabsorción \\
\hline \multirow[t]{2}{*}{ Jul-09 } & 1 & $22,1 \pm 3,8$ & 90 & Crecimiento \\
\hline & 2 & $38,0 \pm 9,3$ & 445 & Madurez \\
\hline Ago-09 & 1 & $33,0 \pm 6,3$ & 702 & Madurez \\
\hline \multirow[t]{2}{*}{ Sep-09 } & 1 & $25,8 \pm 4,9$ & 169 & Crecimiento \\
\hline & 2 & $36,8 \pm 4,6$ & 134 & Madurez \\
\hline \multirow[t]{2}{*}{ Oct-09 } & 1 & $21,9 \pm 3,7$ & 45 & Crecimiento \\
\hline & 2 & $41,3 \pm 4,6$ & 83 & Reabsorción \\
\hline Nov-09 & 1 & $24,2 \pm 9,1$ & 158 & Crecimiento \\
\hline Dic-09 & 1 & $14,5 \pm 9,4$ & 18 & Crecimiento \\
\hline \multirow[t]{2}{*}{ Ene-10 } & 1 & $15,6 \pm 3,2$ & 228 & Crecimiento \\
\hline & 2 & $38,1 \pm 3,8$ & 42 & Madurez \\
\hline \multirow[t]{2}{*}{ Feb-10 } & 1 & $14,1 \pm 2,4$ & 54 & Crecimiento \\
\hline & & $>12$ & & Inicio de gametogénesis* \\
\hline
\end{tabular}

${ }^{*}$ Los ovocitos con diámetros inferiores a $12 \mu \mathrm{m}$ no presentaron un citoplasma desarrollado por lo que se les consideró como tempranos

\section{Crecimiento de Los OVocitos}

Las tasas de crecimiento ovocitario mostraron un valor promedio de $0,41 \pm 0,10 \mu \mathrm{m} \mathrm{día}^{-1}$, con un valor máximo de $0,67 \mu$ día $^{-1}$ entre septiembre y octubre y un valor mínimo de $0,29 \mu$ día $^{-1}$ entre octubre y noviembre 2009 (Tabla 2).

\section{FASES REPRODUCTIVAS}

Las observaciones citológicas hicieron posible identificar 5 estadios de desarrollo gametogénico: gametogénesis temprana, crecimiento, madurez, desove y reabsorción (Fig. 3; hembras A-E y machos F- J). Se identificaron las características histológicas en los diferentes estadios de reproducción de las hembras y de los machos (Tabla 3).

El porcentaje de ocupación gonadal correspondiente a cada estadio de desarrollo, mostró que de marzo a septiembre, el proceso de reproducción se mantuvo con poca variación. La adición de los ovocitos en crecimiento y maduros, ocuparon el mayor porcentaje de la gónada con 92\%; el resto estuvo ocupado por ovocitos que presentaron una talla correspondiente a aquella observada en ovocitos en absorción. De octubre 2009 a febrero 2010, la gónada estuvo ocupada predominantemente por ovocitos en inicio de gametogénesis y en crecimiento (75\%), así como por ovocitos en fase de madurez (25\%). En este periodo los organismos se prepararon para el inicio de un nuevo ciclo gametogénico (Fig. 4).

Tabla 2. Tasa de crecimiento ovocitaria de Chione fluctifraga para cada cohorte (*modelo de regresión lineal significativo) / Oocyte growth rate for each cohort of Chione fluctifraga (*significant linear regression)

\begin{tabular}{cccccc}
\hline Cohorte & $\begin{array}{c}\text { Duración } \\
\text { (días) }\end{array}$ & Ecuación & $\mathrm{R}^{2}$ & $\begin{array}{c}\text { Tasa de crecimiento } \\
\left(\mu \mathrm{m} \mathrm{dia}^{-1}\right)\end{array}$ & $P$-valor \\
\hline 1 & 37 & $\mathrm{y}=0,3514 \mathrm{x}+36.000$ & 1 & 0,35 & --- \\
2 & 66 & $\mathrm{y}=0,3682 \mathrm{x}+23.956$ & 0,9969 & 0,37 & $0,0354^{*}$ \\
3 & 61 & $\mathrm{y}=0,4425 \mathrm{x}+24.457$ & 0,9999 & 0,44 & $0,0044^{*}$ \\
4 & 62 & $\mathrm{y}=0,4031 \mathrm{x}+23.802$ & 0,9998 & 0,40 & $0,0089^{*}$ \\
5 & 66 & $\mathrm{y}=0,4464 \mathrm{x}+19.714$ & 0,9264 & 0,45 & 0,1748 \\
6 & 65 & $\mathrm{y}=0,3409 \mathrm{x}+24.355$ & 0,9980 & 0,34 & $0,0283^{*}$ \\
7 & 50 & $\mathrm{y}=0,4008 \mathrm{x}+25.779$ & 0,9925 & 0,40 & 0,0550 \\
8 & 21 & $\mathrm{y}=0,6714 \mathrm{x}+24.400$ & 1 & 0,67 & --- \\
9 & 42 & $\mathrm{y}=0,2905 \mathrm{x}+22.400$ & 1 & 0,29 & -- \\
10 & 27 & $\mathrm{y}=0,4111 \mathrm{x}+22.200$ & 1 & 0,41 & -- \\
11 & 59 & $\mathrm{y}=0,4972 \mathrm{x}+7.378$ & 0,9733 & 0,50 & 0,1044 \\
\hline
\end{tabular}



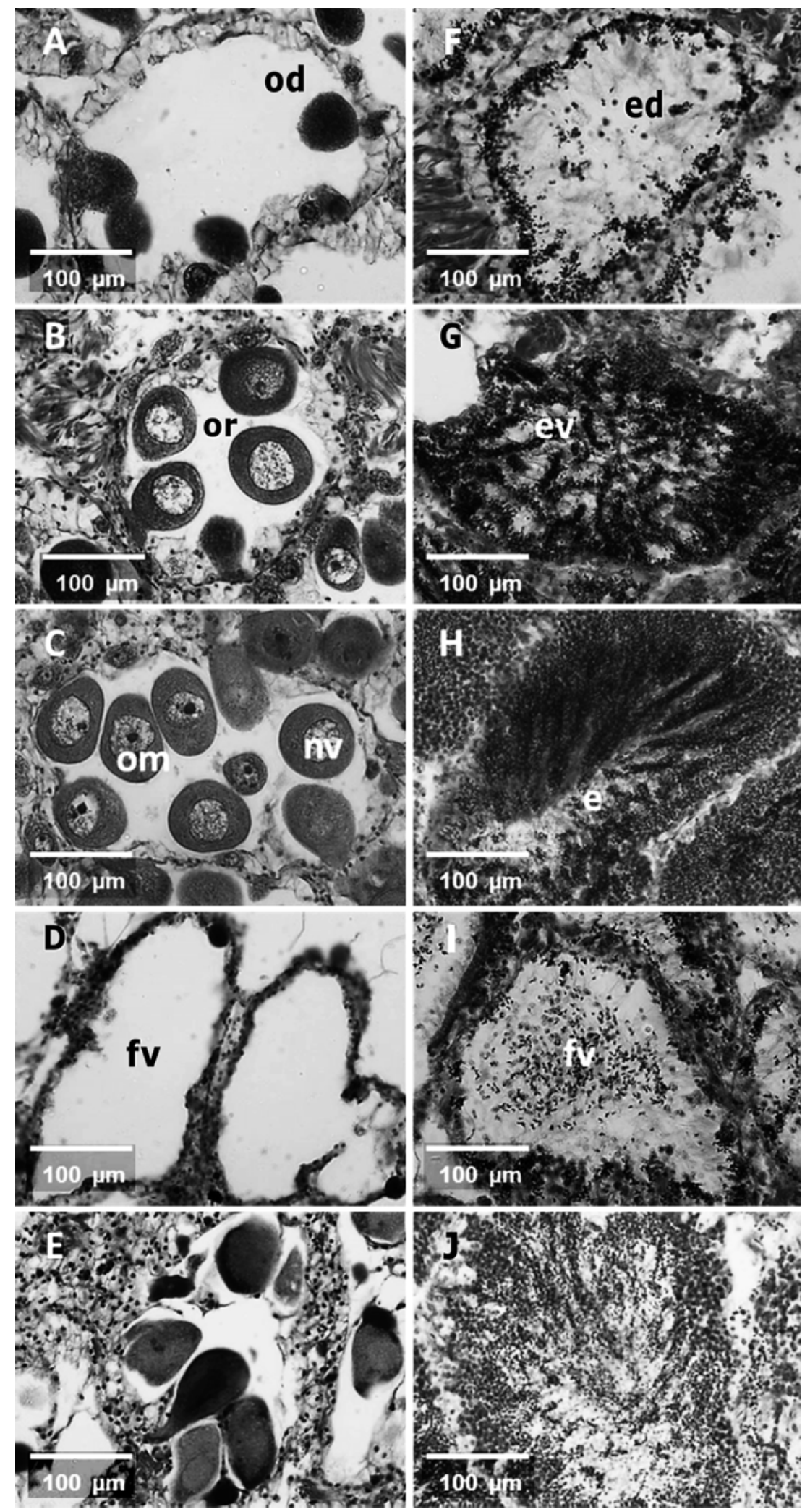

Figura 3. Estadios de desarrollo gonádico de Chione fluctifraga en función de las características citológicas de la gónada. Hembras: (A) gametogénesis temprana, (B) crecimiento, (C) madurez, (D) desove, (E) reabsorción. Machos: (F) gametogénesis temprana, (G) crecimiento, (H) madurez, (I) desove, $(J)$ reabsorción. e = espermatocito, ed = espermatocito en desarrollo, ev = espermatocitos visibles, $\mathrm{fv}=$ folículo vacío, $\mathrm{nv}=$ núcleo visible, od $=$ ovocito en desarrollo, om = ovocito maduro, or = ovocito de forma regular / Stages of gonadal development in Chione fluctifraga, based on cytological characteristics of the gonad. Females: (A) early gametogenesis, (B) growth, (C) mature, (D) spawned, (E) resorption. Males: $(F)$ early gametogenesis, $(G)$ growth, $(H)$ mature, (I) spawned, (J) resorption. e = spermatocyte, ed = developing spermatocyte, ev = visible spermatocytes, $\mathrm{fv}=$ empty follicle, $\mathrm{nv}=$ visible nucleus, $\mathrm{od}=$ developing oocyte, om = mature oocyte, or = regular-shaped oocyte 


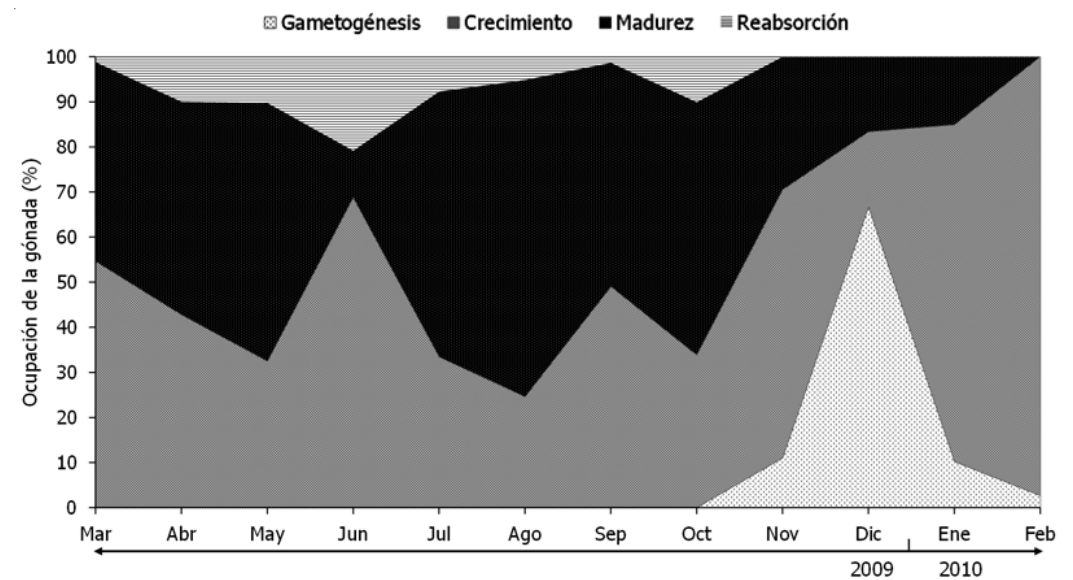

Figura 4. Porcentaje de ocupación de la gónada correspondiente a cada estadio de desarrollo de Chione fluctifraga / Occupancy of the gonad for each stage of development of Chione fluctifraga

Tabla 3. Características histológicas de los diferentes estadios gametogénicos en hembras y machos de Chione fluctifraga / Histological characteristics of the different gametogenic stages in females and males of Chione fluctifraga

\begin{tabular}{|c|c|c|}
\hline Estadio gametogénico & Hembras & Machos \\
\hline Gametogénesis temprana & $\begin{array}{l}\text { La pared de los acinos se observa } \\
\text { engrosada, con gran cantidad de } \\
\text { ovogonias y con algunos ovocitos en } \\
\text { desarrollo, ausencia de tejido folicular y } \\
\text { presencia amplia de tejido conectivo } \\
\text { interfolicular. }\end{array}$ & $\begin{array}{l}\text { Folículos pequeños y dispersos conteniendo } \\
\text { células de afinidad basófila presumiblemente } \\
\text { espermatocitos en desarrollo, tejido conectivo } \\
\text { interfolicular. }\end{array}$ \\
\hline Crecimiento & $\begin{array}{l}\text { El espacio folicular se ve ocupado por } \\
\text { múltiples ovocitos de formas regulares y } \\
\text { diferentes tamaños (en desarrollo) siendo } \\
\text { los más comunes los previtelogénicos, } \\
\text { pared folicular engruesada y ausencia de } \\
\text { tejido conectivo interfolicular. }\end{array}$ & $\begin{array}{l}\text { Aumento de tamaño en los folículos y } \\
\text { reducción del tejido conectivo interfolicular, } \\
\text { espermatocitos visibles. }\end{array}$ \\
\hline Madurez & $\begin{array}{l}\text { Ovocitos grandes de formas oval o } \\
\text { circular dentro del folículo (diámetro } \\
\sim 35 \mu \mathrm{m} \text { ), todos con núcleo visible, } \\
\text { en algunos se apreció el nucléolo, } \\
\text { pared folicular reducida y se detectó } \\
\text { poco tejido conectivo interfolicular. }\end{array}$ & $\begin{array}{l}\text { Folículos ampliamente desarrollados con } \\
\text { arreglo centrífugo más denso en la } \\
\text { periferia y menos denso en el centro, los } \\
\text { espermatozoides fueron visibles por sus } \\
\text { colas alineadas al centro del folículo, } \\
\text { tejido conectivo interfolicular reducido. }\end{array}$ \\
\hline Desove & $\begin{array}{l}\text { Folículos vacíos con algunos ovocitos } \\
\text { pedunculados. }\end{array}$ & $\begin{array}{l}\text { Folículos vacíos, con presencia de } \\
\text { escasos espermatozoides en su interior } \\
\text { alineados hacia el centro, espacios vacíos } \\
\text { entre presencia de tejido conectivo } \\
\text { interfolicular. }\end{array}$ \\
\hline Reabsorción & $\begin{array}{l}\text { Presencia de ovocitos de gran tamaño } \\
\text { con bordes difusos (lisis) dentro } \\
\text { del folículo, paredes del folículo } \\
\text { engrosadas, aparición de tejido conectivo } \\
\text { interfolicular, y en algunos casos se } \\
\text { detectan amebocitos. }\end{array}$ & $\begin{array}{l}\text { Folículos con su interior difuso, no } \\
\text { se identificaron espermatozoides ni } \\
\text { espermatocitos, aumento del tejido } \\
\text { conectivo interfolicular. }\end{array}$ \\
\hline
\end{tabular}




\section{Proporción de SeXos}

De 180 individuos muestreados, 76 (42\%) fueron indiferenciados (ausencia de tejido gonádico), 58 (32\%) machos, y 46 (26\%) hembras. La proporción sexual $(1,3 \mathrm{M}: 1 \mathrm{H}, \mathrm{n}=104)$ no difirió significativamente de la proporción esperada de 1:1 $(P>0,05)$. No se encontraron organismos hermafroditas.

\section{ÍNDICE DE CONDICIÓN}

El promedio del índice de condición mostró 2 máximos en el ciclo anual; en agosto (29) y en diciembre (26) (Fig. 5). No obstante, en abril decreció a 20, cuando la temperatura y la clorofila fueron de las más altas registradas (Fig. 6). El análisis de varianza arrojó diferencias significativas ( $P=0,0002)$ en julio, agosto, septiembre, octubre y noviembre respecto a los demás meses para un nivel de confianza del 95\%.

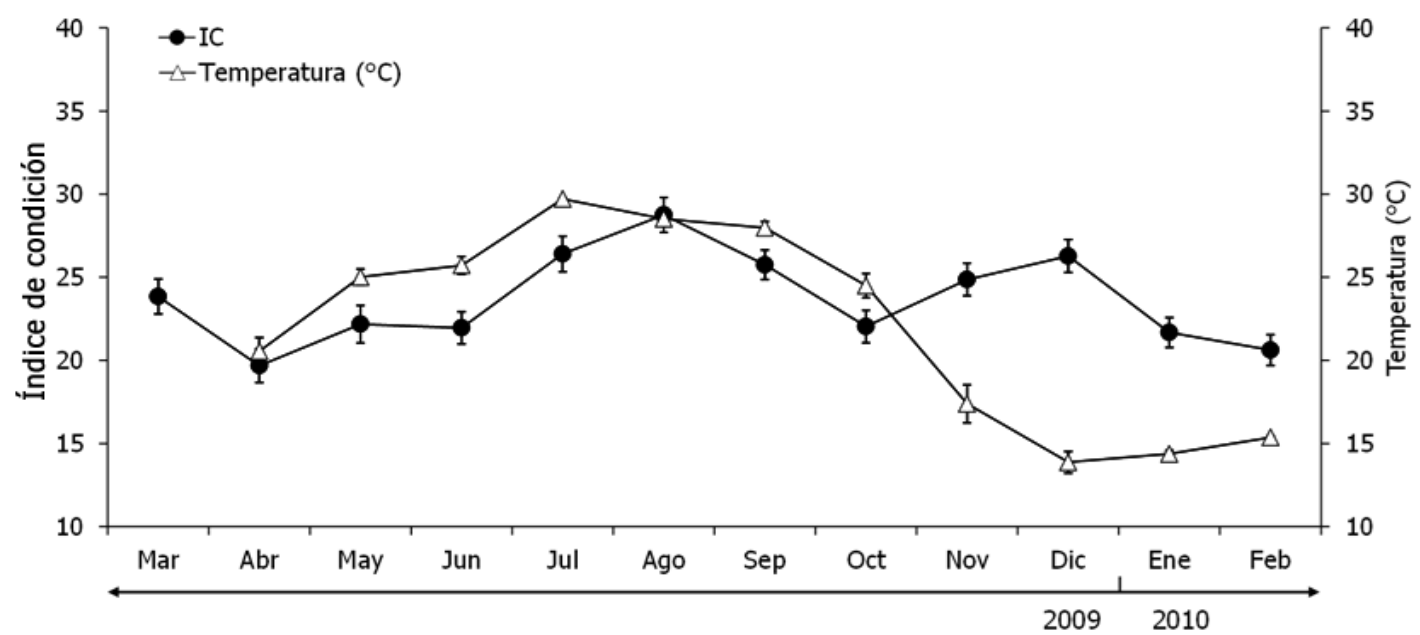

Figura 5. Variación mensual del índice de condición (IC \pm desviación estándar) de Chione fluctifraga y registros de temperatura del agua de mar/ Monthly variation of condition index (IC \pm standard deviation) of Chione fluctifraga and seawater temperature

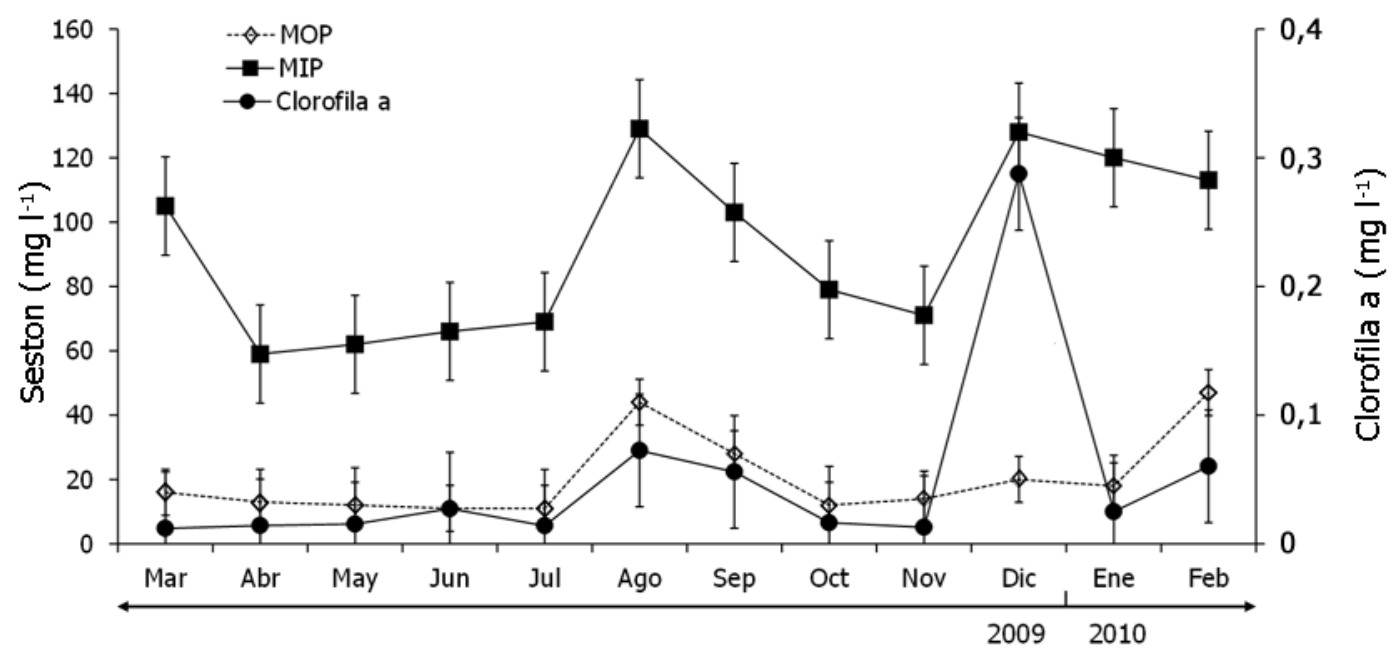

Figura 6. Concentraciones de materia orgánica particulada (MOP), materia inorgánica particulada (MIP) y clorofila $a$ en Bahía San Jorge (Sonora, México) / Concentrations of particulate organic matter (MOP), particulate inorganic matter (MIP), and chlorophyll $a$ at Bahía San Jorge (Sonora, Mexico) 


\section{Parámetros ambientales}

La temperatura del agua tuvo un valor promedio máximo de $30^{\circ} \mathrm{C}$ en julio y el menor de $14^{\circ} \mathrm{C}$ en diciembre (Fig. 5). La clorofila $a$ tuvo escasa variación de abril a diciembre $\left(0,3 \pm 0,1 \mu \mathrm{g} \mathrm{l}^{-1}\right)$, y se observó un pico de 2,9 $\mu \mathrm{g} \mathrm{l}^{-1}$ en enero. El material orgánico particulado (MOP) permaneció relativamente constante con intervalo de $11-28 \mathrm{mg} \mathrm{l}^{-1}$ en el ciclo anual, excepto en septiembre donde se observó un valor de $44 \mathrm{mg} \mathrm{l}^{-1}$. El material inorgánico particulado (MIP) presentó 3 picos: en abril $105 \mathrm{mg} \mathrm{l}^{-1}$, en septiembre $129 \mathrm{mg} \mathrm{l}^{-1} \mathrm{y}$ en enero $128 \mathrm{mg} \mathrm{l}^{-1}$ (Fig. 6). Como referencia, los valores más bajos de la relación MIP/MOP (mayor disponibilidad de material orgánico) se observó en mayo con 4,5, en septiembre con 2,9 y en diciembre con 5,0; la salinidad tuvo un intervalo de 36-42 registrándose el valor más alto en abril y el más bajo en septiembre.

\section{Discusión}

El presente estudio es el primero que se lleva a cabo sobre la biología reproductiva de poblaciones naturales explotadas de la almeja negra Chione fluctifraga. Este tipo de estudios son importantes ya que generan información científica sólida para aplicarse en el uso y manejo de una especie de importancia comercial.

Los resultados mostraron que $C$. fluctifraga tiene un ciclo reproductivo semianual, caracterizado por el desarrollo de varias cohortes ovocitarias así como desoves continuos. La gametogénesis en la especie es un proceso continuo de abril a diciembre. En este período se observan machos en todos los estadios gametogénicos. Lo anterior ha sido observado en otros bivalvos (Baqueiro \& Stuardo 1977, Baqueiro et al. 1982, Baqueiro \& Masso 1988, Avellanal et al. 2002). Las observaciones histológicas y el rango asociado al diámetro de los ovocitos, aportaron evidencia de que el ciclo gametogénico se inició en marzo con la primera cohorte, de acuerdo con esto, la distribución de frecuencias del diámetro de ovocitos mostró su mayor actividad reproductiva con ovocitos en etapa de crecimiento (22,224,3 $\mu \mathrm{m}$ ), maduración (36-38 $\mu \mathrm{m}$ ), y reabsorción (46-49 $\mu \mathrm{m})$ de marzo a octubre cuando la temperatura fue mayor a $25^{\circ} \mathrm{C}$ y se detectaron los valores máximos de MOP. Estos resultados son similares a los reportados por Hmida et al. (2010) para Solen marginatus (navaja) donde el intervalo en los diámetros de los ovocitos en la etapa de crecimiento era de 15-25 $\mu$ m y en maduración de 25-40 $\mu \mathrm{m}$. Durante la fase de mayor actividad reproductiva las proporciones de ovocitos en crecimiento (54,6\%) y maduración (44,2\%) permanecieron sin mucha variación hasta noviembre. La actividad reproductiva disminuyó considerablemente a partir de noviembre cuando se observaron las temperaturas más bajas y las tallas menores de los ovocitos. Las cohortes que se presentaron a partir de diciembre, estuvieron representadas por ovocitos en inicio de gametogénesis $(66,7 \%)$ y ovocitos en crecimiento en enero $(74,7 \%)$ y febrero $(97,4 \%)$, lo que hace suponer que a partir de diciembre los organismos se encontraban en el inicio de un nuevo ciclo gametogénico. Este patrón de reproducción de $C$. fluctifraga es similar al de $C$. californiensis en Bahía Magdalena, B.C.S. (México), donde la gametogénesis fue continua desde abril hasta diciembre, y al igual que en este estudio la actividad reproductiva se interrumpió en invierno (GarcíaDomínguez et al. 1993).

Los resultados del análisis modal indicaron que durante la gametogénesis de $C$. fluctifraga se presentaron de manera continua 2 modas que correspondieron a estadios de crecimiento y madurez, junio fue la excepción presentando 3 modas, 2 en crecimiento y una en reabsorción, lo que hace suponer que en este período se presentó el desove más intenso. Lo anterior muestra que en esta especie existe una continua generación de cohortes ovocitarias a través del proceso de gametogénesis durante el ciclo reproductivo, como también ha sido observado en Ruditapes decussatus, $R$. philippinarum (Laruelle et al. 1994), y Pecten maximus (Paulet \& Boucher 1991, Saout 2000). La diferencia con estos estudios es que en $C$. fluctifraga los ovocitos maduros son liberados continuamente a lo largo de 9 meses del año, mientras que en estas especies los ovocitos son liberados en uno o 2 desoves que ocurren durante un período relativamente fijo del año.

La ausencia de organismos hermafroditas en las cohortes estudiadas, sugiere que la especie es gonocórica con predominancia de machos. Algunos autores señalan que en las poblaciones donde la proporción sexual favorece a los machos, los organismos se encuentran gran parte del tiempo en condiciones poco favorables para la reproducción, pero cuando las condiciones son propicias, la proporción de hembras aumenta rápidamente (Thielley 1993, Lango-Reynoso 1999). Las almejas de este estudio habitan principalmente en la zona intermareal, con prolongados períodos de tiempo de desecación, lo que podría generar situaciones de estrés. Este aspecto sería ejemplo en las hembras de la ostra perlera Pinctada margaritifera las cuales son muy sensibles al estrés y su número disminuye considerablemente cuando se presentan valores extremos de temperatura, ausencia de 
alimento, contaminación, exposición a manejo (cultivos), etc. (Chávez-Villalba et al. 2011). Al parecer, las hembras de $C$. fluctifraga serían menos sensibles al estrés debido a que estuvieron presentes todo el año, aumentando su proporción en la época reproductiva, pero disminuyendo en invierno cuando no se reproducen. Esto coincide con lo encontrado para Crassostrea gigas en la misma región (Chávez-Villalba et al. 2007). Así también, los resultados mostraron altas proporciones de organismos indiferenciados. De acuerdo con algunos autores, algunas especies de venéridos pasan por situaciones que los obligan a reabsorber el material reproductivo residual (García-Domínguez et al. 1993, Villalejo-Fuerte \& CeballosVázquez 1996). Otros indican que los individuos indiferenciados pueden ser una fase de transición entre macho y hembra o viceversa (Pouvreau et al. 2000). Estos autores resaltan la dificultad de hallar hermafroditas entre las ostras perleras (P. margaritifera), de 3360 analizadas sólo encontraron 7 individuos bisexuales. Por lo tanto, se requieren más estudios que determinen si entre el gran número de individuos indiferenciados no se oculta la presencia de hermafroditas en esta especie.

El análisis cualitativo de las gónadas (características citológicas) permitió identificar 5 fases reproductivas en hembras y machos: (1) gametogénesis temprana, (2) crecimiento, (3) maduración, (4) desove y (5) reabsorción de gametos. En las hembras, estas fases se asociaron a través de un examen cuantitativo (conteo y medición de ovocitos) a la talla de sus ovocitos. Este tipo de análisis ha permitido establecer escalas reproductivas basadas en el diámetro de los ovocitos y observaciones histológicas de la gónada para otras especies de bivalvos como Crassostrea gigas (Lango-Reynoso et al. 2000), Atrina maura (Enríquez-Díaz et al. 2002), y Solen marginatus (Hmida et al. 2010). En este trabajo se propone una escala de reproducción para Chione fluctifraga bajo los criterios mencionados, la cual podría servir de referencia para estudios posteriores para la especie y posiblemente para almejas del mismo género.

La influencia de las variables ambientales en el proceso reproductivo ha sido tratada en numerosos estudios desde hace décadas (López et al. 2005). En los bivalvos, las variables con mayor influencia son la temperatura y la cantidad de alimento disponible (Loosanoff \& Davis 1963, Giese \& Kanatani 1987, Barber \& Blake 1991, Darriba 2001). El inicio de la actividad reproductiva ocurrió cuando la temperatura del agua fue $>20^{\circ} \mathrm{C}$ (marzo) con alta disponibilidad de alimento indicada (MIP/MOP 6,6). El periodo de mayor actividad reproductiva (primavera- verano) coincidió también con valores altos de temperatura $\left(>26^{\circ} \mathrm{C}\right)$ y con los incrementos (36/116 $\mathrm{mg} \mathrm{l}^{-1}$ ) de MOP y MIP, respectivamente. Lo anterior coincide con lo encontrado por Chávez-Villalba et al. (2005) para C. gigas quienes observaron una relación entre alimentación y actividad reproductiva durante primavera-verano. De acuerdo con Delgado \& PérezCamacho (2005), en Ruditapes decussatus la energía procedente de la ingestión se destina primordialmente al proceso de reproducción, de esta forma, el alimento disponible guarda relación directa con el desarrollo gonadal y por lo tanto con la velocidad de desarrollo de los gametos. Durante el periodo reproductivo de $C$. fluctifraga la tasa de crecimiento ovocitaria se mantuvo relativamente constante (promedio $0,44 \mu^{-1}$ ) debido probablemente a condiciones favorables de temperatura $\left(>20^{\circ} \mathrm{C}\right.$ ) y de disponibilidad de alimento. Lo anterior implica ventajas para la especie ya que se maximiza el rendimiento reproductivo (fecundidad) con más gametos maduros listos para ser liberados al medio.

En algunas especies, el desove se inicia cuando la temperatura excede de un nivel crítico característico (Maeda-Martínez 2001). El inicio de los desoves en $C$. fluctifraga se detectó en abril cuando la temperatura del agua llegó a $21^{\circ} \mathrm{C}$, pero al parecer el desove principal ocurrió durante julio-agosto (2009) cuando la temperatura alcanzó sus máximos valores $\left(29-30^{\circ} \mathrm{C}\right)$. Por ejemplo, en Chione californiensis el desove se presentó cuando la temperatura fue mayor a $24^{\circ} \mathrm{C}$ (García-Domínguez et al. 1993), mientras que el desove de Saccostrea palmula en Bahía Magdalena (México) inició a partir de los 21,5 $\mathrm{C}$ (Romo-Piñera 2005). Al parecer este fenómeno podría ser común, pues también ha sido observado en otras especies y venéridos como Mercenaria mercenaria (MacDonald \& Thompson 1985, Manzi et al. 1985, Pearse et al. 1991, Brey \& Hain 1992). En invierno, se observó una fase de reposo reproductivo probablemente debido a que las temperaturas registradas en este periodo fueron menores a $20^{\circ} \mathrm{C}$. La evidencia indica que los ovocitos observados en diciembre y enero en fase de madurez, no se encontraron en febrero probablemente porque fueron reabsorbidos en la gónada. En enero y febrero, aún con bajas temperaturas, sólo se detectaron ovocitos en inicio de gametogénesis y en crecimiento, y permanecieron así hasta que re-inició el ciclo de reproducción con el incremento de la temperatura del agua en marzo. Estas observaciones sugieren que la gametogénesis en $C$. fluctifraga está directamente relacionada con la variación de la temperatura del agua, a diferencia de especies del 
mismo género como C. undatella en B.C.S. y C. cancellata en Florida, las cuales presentaron periodos reproductivos continuos a lo largo de todo el año sin efecto aparente de la variación estacional de la temperatura (Moore \& López 1969, Baqueiro \& Masso 1988).

El índice de condición (IC) es reconocido por su importancia para evaluar el estado nutricional de los bivalvos, su calidad comercial, así como conocer de manera general su ciclo de reproducción (Bodoy et al. 1986, Crosby \& Gale 1990, Mason \& Nell 1995, Baghurst \& Mitchell 2002). Por ejemplo, el IC para R. decussatus y R. philippinarum en Brest, Francia, mostró que los valores se incrementan desde marzo hasta finales de mayo, después se produce una disminución del índice y vuelve a incrementarse hasta mediados de agosto, y posteriormente a principios de septiembre se produce otra disminución importante coincidiendo con el desove de finales de verano (Laruelle et al. 1994). Este comportamiento es muy similar al observado para $C$. fluctifraga, donde los valores del IC se incrementaron desde marzo hasta agosto con descensos en abril, junio y, el más importante, en septiembre. Esto, además de coincidir en gran medida con la variación del MOP, sugiere una fuerte relación del IC con el ciclo de reproducción; el valor más alto se detectó durante la mayor actividad reproductiva en verano y el desove principal se puede asociar con el posterior decremento significativo del IC en septiembre. No obstante, el otro pico del IC en diciembre puede asociarse a un almacenamiento de nutrientes a partir de un incremento de la masa fitoplanctónica durante ese mes. Esto indica que la especie podría tener una estrategia de reproducción conservadora, donde las reservas de energía se acumulan y después son movilizadas para soportar la producción de gametos (Ren et al. 2003). Esto se ha observado también en otros bivalvos de la zona como Argopecten circularis (Villalejo-Fuerte \& CeballosVázquez 1996) y de otras latitudes como C. gigas (Li et al. 2009).

Los resultados de este estudio muestran que el ciclo de reproducción de $C$. fluctifraga está regulado principalmente por la temperatura, siendo de manera continua de marzo a noviembre cuando los valores son mayores a $20^{\circ} \mathrm{C}$. Durante este periodo se observa el desarrollo de cohortes de ovocitos que maduran y son liberados al medio en desoves continuos pero con un evento principal en verano asociado con la máxima temperatura del agua. La disponibilidad de alimento durante los periodos de reproducción y de reposo sexual contribuye con el desarrollo de los gametos y con el almacenamiento de nutrientes, respectivamente, sugiriendo una estrategia de reproducción conservadora. Los resultados del IC permiten identificar los periodos donde las almejas presentan mayor rendimiento en carne, lo que podría servir a los productores para la planeación de sus cosechas. La escala reproductiva propuesta podría asistir en futuros estudios de la especie u otras similares. Esta información puede ser útil para contribuir a regular la pesquería de $C$. fluctifraga, aunque es necesario realizar estudios de abundancia y distribución de la especie en las zonas de explotación. Sin embargo, dada la coincidencia con Martínez-Córdova (1988), podemos sugerir disminuir las capturas desde finales de primavera hasta agosto cuando existe la mayor actividad reproductiva.

\section{Agradecimientos}

Se agradece a la Cooperativa San Jorge por donar las almejas y apoyar en los muestreos, así como a Andrés Hernández-Ibarra, María R. López-Tapia y Edgar Alcántara-Razo del CIBNOR por su apoyo en las salidas y en los análisis de laboratorio. Los comentarios de tres evaluadores anónimos ayudaron a mejorar la versión original. Ariaana Castillo-Durán recibe una beca de doctorado de CONACyT. Este estudio fue financiado en parte por los proyectos CIBNOR 3.1 y 931-1.

\section{LITERATURA CITADA}

Avellanal MH, E Jaramillo, E Clasing, P Quijón \& H Contreras. 2002. Reproductive cycle of the bivalves Ensis macha (Molina, 1782) (Solenidae), Tagelus dombeii (Lamarck, 1818) (Solecurtidae), and Mulinia edulis (King, 1831) (Mactridae) in southern Chile. The Veliger 45: 33-44.

Baghurst BC \& JG Mitchell. 2002. Sex-specific growth and condition of the Pacific oyster (Crassostrea gigas Thunberg). Aquaculture Research 33: 1253-1263.

Baqueiro E \& JA Masso. 1988. Variaciones poblacionales y reproducción de dos poblaciones de Chione undatella (Sowerby, 1835) bajo diferentes regímenes de pesca en la Bahía de La Paz, B.C.S. México. Ciencia Pesquera 6: 51-67.

Baqueiro E \& J Stuardo. 1977. Observaciones sobre la biología, ecología y explotación de Megapitaria aurantiaca (Sowerby 1831), M. squalida (Sowerby 1835) y Dosinia ponderosa (Gray 1838) (Bivalvia: Veneridae) de la Bahía de Zihuatanejo e Isla Ixtapa, Gro., México. Anales del Centro de Ciencias del Mar y Limnología 4: 161-208. 
Baqueiro CE, M Muciño \& R Merino. 1982. Análisis de una población de pata de mula Anadara tuberculosa sujeta a explotación intensiva en la Bahía de La Paz, Baja California Sur, México. Ciencia Pesquera 3: 75-82.

Barber BJ \& NJ Blake. 1991. Reproductive physiology. In: Shumway SE (ed). Scallops: biology, ecology and aquaculture. Developments in Aquaculture and Fisheries Science 21: 377-428. Elsevier, Amsterdam.

Bhattacharya CG. 1967. A simple method of resolution of a distribution into gaussian components. Biometrics 23: 115135.

Bodoy A, J Prou \& JP Berthome. 1986. Étude comparative de différents indices de condition chez l'huître creuse (Crassostrea gigas). Haliotis 15: 173-182.

Borda CA \& R Cruz. 2004. Reproducción y reclutamiento del molusco Anadara tuberculosa (Sowerby, 1833) en el Pacífico Colombiano. Revista de Investigaciones Marinas 25: 185-195.

Brey T \& S Hain. 1992. Growth, reproduction and production of Lissarca notorcadensis (Bivalvia: Philobrydae) in the Weddell Sea, Antartica. Marine Ecology Progress Series 82: 219-226.

Chávez-Villalba J, J Pommier, J Adriamiseza, S Pouvreau, J Barret, JC Cochard \& M Le Pennec. 2002. Broodstock conditioning of the oyster Crassostrea gigas: origin and temperature effect. Aquaculture 214: 115-130.

Chávez-Villalba J, MR López-Tapia, JM Mazón-Suástegui \& M Robles-Mungaray. 2005. Growth of the oyster Crassostrea corteziensis (Hertlein, 1951) in Sonora, Mexico. Aquaculture Research 36: 1337-1344.

Chávez-Villalba J, F Villelas-Ávila \& C Cáceres-Martínez. 2007. Reproduction, condition and mortality of the Pacific oyster Crassostrea gigas (Thunberg) along coastal Sonora, Mexico. Aquaculture Research 38: 268-278.

Chávez-Villalba J, C Soyez, A Huvet, Y Gueguen, C Lo \& G Le Moullac. 2011. Determination of gender in the pearl oyster Pinctada margaritifera. Journal of Shellfish Research 30: 231-240.

Crosby MP \& LD Gale. 1990. A review and evaluation of bivalve condition index methodologies with a suggested standard method. Journal of Shellfish Research 9: 233-237.

Darriba S. 2001. Biología de la navaja Ensis arcuatus (Jeffreys, 1865) de la Ría de Vigo (NO de España): Crecimiento y reproducción. Tesis doctoral, Universidad de Vigo, Vigo Pontevedra, 283 pp.

Delgado M \& A Pérez-Camacho. 2005. Histological study of the gonadal development of Ruditapes decussatus (L.) (Mollusca: Bivalvia) and its relationship with available food. Scientia Marina 69: 87-97.

Dutertre M, PG Beninger, L Barillé, M Papin, P Rosa, AL Barillé \& J Haure. 2009. Temperature and seston quantity and quality effects on field reproduction of farmed oysters, Crassostrea gigas, in Bourgneuf Bay, France. Aquatic Living Resources 22: 319-329.
Enríquez-Díaz M, C Cáceres-Martínez, J ChávezVillalba, G Le Pennec \& M Le Pennec. 2002. Gametogenesis of Atrina maura (Bivalve: Pinnidae) under artificial conditions. Invertebrate Reproduction and Development 43: 151-161.

Enríquez-Díaz M, S Pouvreau, J Chávez-Villalba \& M Le Pennec. 2009. Gametogenesis, reproductive investment, and spawning behavior of the Pacific giant oyster Crassostrea gigas: evidence of an environment-dependent strategy. Aquaculture International 17: 491-506.

García-Domínguez FA. 2002. Estrategias reproductivas de bivalvos marinos en el noroeste mexicano. Tesis Doctoral, Facultad de Medicina Veterinaria y Zootecnia, Universidad de Colima, Colima, 119 pp.

García-Domínguez F, G García-Melgar \& P GonzálezRamírez. 1993. Ciclo reproductivo de la almeja roñosa, Chione californiensis (Broderip, 1835), en Bahía Magdalena, Baja California Sur, México. Ciencias Marinas 19: 15-28.

Giese A \& H Kanatani. 1987. Maduration and spawning. In: Giese AC, J Pearse \& V Pearse (eds). Reproduction of Marine Invertebrates 9: 251-329. Blackwell Scientific and Boxwood, San Diego.

Gribben PE, J Helson \& AG Jeffs. 2004. Reproduction cycle of the New Zealand geoduck, Panopea zelandica, in two North Island populations. The Veliger 47: 53-65.

Gutiérrez-Vázquez I. 2007. Maduración y obtención de semilla de la almeja negra, Chione fluctifraga (Sowerby, 1853), Bivalvia: Veneridae, de las costas de Sonora, México. Tesis Maestría, Universidad de Sonora, Hermosillo, 150 pp.

Heffernan PB \& RL Walker. 1989. Quantitative image analysis methods for use in histological studies of bivalve reproduction. Journal of Molluscan Studies 55: 135-137.

Hmida L, N Ayache, Z Haouas \& MS Romdhane. 2010. Oocyte cohort analysis: criteria for an evaluation of the reproductive cycle in Solen marginatus (Pennant, 1777), (Bivalvia: Solenacea) in southern Tunisia. Journal of Shellfish Research 29: 129-134.

Howard DW \& CS Smith. 1983. Histological techniques for marine bivalve mollusks NOAA Technical Memorandum NMFS-F/NEC 25: 1-97.

Lango-Reynoso F. 1999. Détermination de la sexualité chez l'huître Crassostrea gigas (Thunberg, 1793). Tesis Doctoral, Université de Bretagne Occidentale, Brest, 176 pp.

Lango-Reynoso F, J Chávez-Villalba, JC Cochard \& M Le-Pennec. 2000. Oocyte size, a means to evaluate the gametogenic development of the Pacific oyster, Crassostrea gigas (Thunberg). Aquaculture 190: 183-199.

Lango-Reynoso F, J Chávez-Villaba \& M Le Pennec. 2006. Reproductive patterns of the Pacific oyster Crassostrea gigas in France. Invertebrate Reproduction and Development 49: 41-50.

Laruelle F, J Guillou \& YM Paulet. 1994. Reproductive pattern of the clams, Ruditapes decussatus and $R$. philippinarum on intertidal flats in Brittany. Journal of the Marine Biological Association of the United Kingdom 74: 351-366. 
Li Y, JG Qin, X Li \& K Benkendorff. 2009. Monthly variation of condition index, energy reserves and antibacterial activity in Pacific oysters, Crassostrea gigas, in Stansbury (South Australia). Aquaculture 286: 64-71.

Lista M, A Prieto, C Velázquez, C Lodeiros \& G Hernández. 2008. Variación mensual del índice de condición y madurez sexual en la pepitona Arca zebra en el banco de Chacopata, península de Araya, estado Sucre, Venezuela. Saber, Venezuela 20: 29-38.

Loosanoff V \& H Davis. 1963. Rearing of bivalve mollusks. Advances in Marine Biology 1: 1-136.

López J, C Rodríguez \& JF Carrasco. 2005. Comparación del ciclo reproductor de Solen marginatus (Pultney 1799) (Mollusca: Bivalvia) en las rías del Eo y Villaviciosa (Asturias, noroeste de España): relación con las variables ambientales. Boletín del Instituto Español de Oceanografía 21: 317-327.

Lucas A \& P Beninger. 1985. The use of physiological condition indices in marine bivalve aquaculture. Aquaculture 44: 187-200.

MacDonald BA \& RJ Thompson. 1985. Influence of temperature and food availability on the ecological energetics of the giant scallop Placopecten magellanicus. II. Reproductive output and total production. Marine Ecology Progress Series 25: 295-303.

Maeda-Martínez AN. 2001. Los moluscos pectínidos de Iberoamérica: ciencia y acuicultura, 501 pp. Editorial Limusa, México.

Manzi JJ, MY Bobo \& VG Burrell. 1985. Gametogenesis in a population of the hard clam, Mercenaria mercenaria (Linnaeus), in North Santee Bay, South Carolina. The Veliger 28: 186-194.

Martínez-Córdova LR. 1988. Bioecología de la almeja negra Chione fluctifraga (Sowerby, 1853). Revista de Biología Tropical 36: 213-219.

Martínez-Córdova LR. 1996. Contribution to the knowledge of the malacological fauna of four costal lagoons in the state of Sonora, Mexico. Ciencias Marinas 22: 191-203.

Mason CJ \& JA Nell. 1995. Condition index and chemical composition of meats of Sydney rock oysters (Saccostrea commercialis) and Pacific oyster (Crassostrea gigas) at four sites in Port Stephens, NSW. Marine Freshwater Research 46: 873-881.

Metaxatos A. 2004. Population dynamics of the venerid bivalve Callista chione (L.) in a coastal area of the eastern Mediterranean. Journal of Sea Research 52: 293-305.

Moore HV \& NN López. 1969. The ecology of Chione cancellata. Bulletin of Marine Science 19: 131-148.

Morvan C \& AD Ansell. 1988. Stereological methods applied to reproductive cycle of Tapes rhomboides. Marine Biology 97: 355-364.
Moura P, MB Gaspar \& CC Monteiro. 2008. Gametogenic cycle of the smooth clam Callista chione on the southwestern coast of Portugal. Journal of the Marine Biological Association of the United Kingdom 88: 161-167.

Muranaka MS \& JE Lannan. 1984. Broodstock management of Crassostrea gigas: environmental influences on broodstock conditioning. Aquaculture 39: 217-228.

Oyarzún-Cabañas P, JE Toro, R Jaramillo, R Guiñez, C Briones \& M Astorga. 2010. Análisis comparativo del ciclo gametogénico de Perumytilus purpuratus (Bivalvia: Mytilidae), en las localidades de Taltal y Huasco, norte de Chile. Revista de Biología Marina y Oceanografía 45: 4358.

Parsons R, M Takahashi \& B Margrave. 1984. Biological oceanographic processes, 330 pp. Pergamon Press, London.

Paulet YM \& J Boucher. 1991. Is reproduction mainly regulated by temperature or photoperiod in Pecten maximus? International Journal of Invertebrate Reproduction and Development 19: 61-70.

Pearse JS, JB McClintock \& I Bosch. 1991. Reproduction of Antarctic benthic marine invertebrates: tempos, modes and timing. American Zoologist 31: 65-80.

Pouvreau S, A Gangnery, J Tiapari, F Lagarde, M Garnier \& A Bodoy. 2000. Gametogenic cycle and reproductive effort of the tropical blacklip pearl oyster, Pinctada margaritifera (Bivalvia: Pteriidae), cultivated in Takapoto Atoll (French Polynesia). Aquatic Living Resources 13: 37-48.

Ren JS, ID Marsden, AH Ross \& DR Schiel. 2003. Seasonal variation in the reproductive activity and biochemical composition of the Pacific oyster (Crassostrea gigas) from the Marlborough Sounds, New Zealand. New Zealand Journal of Marine \& Freshwater Research 37: 171-182.

Romo-Piñera AK. 2005. Ciclo reproductivo del ostión de mangle Saccostrea palmula (Carpenter, 1857), en Bahía Magdalena, Baja California Sur. Tesis de Maestría, Centro Interdisciplinario de Ciencias Marinas, Instituto Politécnico Nacional, La Paz, México, 74 pp.

Saout C. 2000. Contrôle de la reproduction chez Pecten maximus (L.): Études in situ et expérimentales. Tesis Doctoral, Université de Bretagne Occidentale, Brest, 172 pp.

Sokolowski A, AS Bawazir, E Sokolowska \& M Wolowicz. 2010. Seasonal variation in the reproductive activity, physiological condition and biochemical components of the brown mussel Perna perna from the coastal waters of Yemen (Gulf of Aden). Aquatic Living Resources 23: 177-186.

Strickland JDH \& TR Parson. 1972. A practical handbook of seawater analysis. Fisheries Research of Board Canada, Bulletin 167: 1-311. 
Thielley M. 1993. Etude cytologique de la gamétogenèse, de la sex-ratio et du cycle de reproduction chez l'huître perlière Pinctada margaritifera (L) var. cummingi (Jameson), (mollusques, bivalves): comparaison avec le cycle de Pinctada maculata (Gould). Tesis Doctoral, Université Française du Pacifique, French Polynesia, 233 pp.

Tinoco-Orta GD \& J Cáceres-Martínez. 2003. Infestation of the clam Chione fluctifraga by the burrowing worm Polydora sp. nov. in laboratory conditions. Journal of Invertebrate Pathology 83: 196-205.
Villalejo-Fuerte M \& B Ceballos-Vázquez. 1996. Variación de los índices de condición general, gonádico y de rendimiento muscular en Argopecten circularis (Bivalvia: Pectinidae). Revista de Biología Tropical 44: 591-594.

Walne PR \& R Mann. 1975. Growth and biochemical composition in Ostrea edulis and Crassostrea gigas. In: Barnes H (ed). Ninth European Marine Biology Symposium, pp. 587-607. Aberdeen University Press, Scotland.

Zar JH. 1996. Biostatistical analysis, 662 pp. Prentice-Hall, Englewood Cliff.

Recibido el 22 de mayo de 2012 y aceptado el 6 de febrero de 2013

Editor Asociado: Karin Lohrmann 